\title{
The clinical outcomes of medical therapies in chronic rhinosinusitis are independent of microbiomic outcomes: a double-blinded, randomised placebo-controlled trial*
}

\author{
Lisa M. Cherian, Ahmed Bassiouni, Clare M. Cooksley, Sarah Vreugde, \\ Peter-John Wormald, Alkis J. Psaltis
}

Department of Surgery - Otolaryngology, Head and Neck Surgery, University of Adelaide, Adelaide, Australia
Rhinology 58: 6, $559-567,2020$

https://doi.org/10.4193/Rhin20.055

*Received for publication:

February 16, 2020

Accepted: April 27, 2020

\begin{abstract}
Background: Oral and topical corticosteroids, and antibiotics form the mainstay medical treatment of chronic rhinosinusitis (CRS). Clinical outcomes vary depending on the chosen therapy, resident microbiome and disease phenotype. We conducted a doubleblinded, placebo-controlled Randomised Controlled Trial (RCT) to investigate effects of medical therapy on clinical outcomes and associated microbiome shifts.
\end{abstract}

Methodology: Fifty eligible patients (CRS with and without polyps) were treated for 3 weeks after randomisation into 3 arms: namely oral prednisolone, topical budesonide irrigations and oral doxycycline; each with appropriate placebo. Clinical scoring and microbiome swabs were performed on enrolment, at treatment completion and 3-weeks post treatment completion. Microbiome analysis was performed using the llumina-MiSeq next generation sequencing platform and QIME-2 pipeline.

Results: Significant improvement in clinical scores was observed in prednisolone and budesonide arms at treatment completion but not with antibiotic. Sub-group analysis showed more pronounced effects in patients with polyposis. Corynebacterium and Staphylococcus species predominated, with variable bacterial relative abundance among different treatments at all time-points. The only significant microbiome finding was an increase in bacterial diversity in topical budesonide group immediately after treatment, which returned to baseline 3-weeks post treatment.

Conclusion: Clinical improvement was significant with oral and topical steroid but not empirical antibiotic. Although there were some associated microbiome changes with the various treatments, we could not ascertain the consistency of these and whether they do have a clinical significance at all.

Key words: doxycycline, microbiome, patient outcome assessment, prednisolone, budesonide

\section{Introduction}

Chronic rhinosinusitis (CRS) is a multi-etiological disease where infection and inflammation co-exist in the sinonasal cavity. The exact triggers and mechanism of inflammation are still unclear, but likely include epithelial barrier dysfunction, immune dysfunction, sinus outflow obstruction caused by anatomical variations, and pathogenic bacterial colonization ${ }^{(1)}$. In particular, there is a growing interest in literature to accurately define the contribution of resident microbiome to the health-versusdisease state of the sinonasal mucosa ${ }^{(2,3)}$. These studies suggest that CRS patients may be exhibiting an overall shift away from a healthy microbiome into a (usually vaguely defined) state of "dysbiosis". It is unclear whether this "dysbiotic state" changes over time and, in particular, how factors such as current medical treatments influence this state ${ }^{(4)}$.

Corticosteroids represent the cornerstone of medical therapy in CRS, with literature providing ample evidence for symptom improvement ${ }^{(5,6)}$. This is through the anti-inflammatory action, mediated by glucocorticoid receptors within the host cells ${ }^{(7)}$. Additionally, corticosteroids also potentially influence inflamma- 
tion by affecting bacterial growth either directly, or through excipients used in their commercial preparation ${ }^{(8)}$. Little research has been performed however, in the sinonasal microbiome (9-11) and especially into the influence of corticosteroids on this microbiome ${ }^{(12-14)}$ and the resultant clinical outcomes.

The role of antibiotics in CRS appears less important and its usefulness remains unclear, with some reporting benefits on short term antibiotics and others showing none ${ }^{(15)}$. Despite this, rhinosinusitis is the most common disease for which antibiotics are prescribed routinely in ambulatory settings ${ }^{(16)}$.

Moreover, oral antibiotics influence microbiome of the gastrointestinal tract affecting the host immune system, at times with deleterious effect, with studies associating this with the development of diseases like type-1 diabetes mellitus, bronchial asthma, and obesity in animal and human studies ${ }^{(17,18)}$. Whilst antibiotic effects on the gut microbiome have been studied extensively, only a few small studies have evaluated its effects on sinonasal microbiome.

This RCT aims to (a) evaluate the effects of oral prednisolone, topical budesonide rinses and oral doxycycline on clinical outcomes in un-operated CRS patients and (b) ascertain their influence on sinonasal microbiome. To the best of our knowledge, this is the first RCT of its kind to assess both outcomes simultaneously.

\section{Materials and methods}

\section{Ethics statement}

An informed, written patient consent was obtained prior to study enrolment, in accordance with the declaration of Helsinki. This study was approved by the Central Adelaide Local Health Network Ethics Committee (HREC/15/TQEH/177).

\section{Study design}

This RCT recruited CRS patients without prior history of sinus surgeries, from the ENT out-patient of a tertiary hospital between December 2016 - 2018. Randomization was performed centrally by the hospital pharmacy clinical trial department using computer-generated table of random permutation of 20 numbers to randomise into permuted blocks of 9 to the 3 different treatment interventions. The treatments (active and placebo) were prepacked before study commencement in a double-blind fashion to ensure concealment. Allocated pack numbers corresponding to the randomization list were then available to dispense to trial participants with the allocation numbers provided to participants in the order of study enrolment. All parties remained blinded until final data analysis.

\section{Study population with inclusion and exclusion criteria} CRS patients diagnosed as per the criteria outlined in the European Position Paper on Rhinosinusitis and Nasal Polyps (EPOS) $2012^{(19)}$ guidelines were included in the study. Patients who
Table 1. Treatment arms.

\begin{tabular}{|c|c|}
\hline $\begin{array}{l}\text { Oral } \\
\text { steroid } \\
(n=17)\end{array}$ & $\begin{array}{l}\text { Oral Prednisolone ( } 25 \mathrm{mg} / \text { day for } 1 \text { week then } 12.5 \mathrm{mg} \text { / } \\
\text { day for } 1 \text { week then } 12.5 \mathrm{mg} \text { every other day for } 1 \\
\text { week) } \\
+200 \mathrm{ml} \text { isotonic saline with water for injection }(2 \mathrm{ml} \\
\text { respules) as placebo delivered intranasally } 2 \text { times a } \\
\text { day } \\
+ \text { Oral placebo for antibiotic } 2 \text { tablets on day one, fol- } \\
\text { lowed by one tablet once daily for } 3 \text { weeks }\end{array}$ \\
\hline $\begin{array}{l}\text { Topical } \\
\text { steroid } \\
(n=17)\end{array}$ & $\begin{array}{l}\text { Placebo for oral steroid in tapering doses for three } \\
\text { weeks } \\
+ \text { Topical Budesonide }(0.5 \mathrm{mg} / 2 \mathrm{ml} \text { respules) washes of } \\
\text { the nasal cavities and sinuses delivered intranasally } 2 \\
\text { times a day in } 200 \mathrm{ml} \text { isotonic saline } \\
+ \text { Oral placebo for antibiotic } 2 \text { tablets on day one, fol- } \\
\text { lowed by one tablet once daily for } 3 \text { weeks }\end{array}$ \\
\hline $\begin{array}{l}\text { Oral } \\
\text { antibiotic } \\
(n=16)\end{array}$ & $\begin{array}{l}\text { Placebo for oral steroid in tapering doses for three } \\
\text { weeks } \\
+200 \mathrm{ml} \text { isotonic saline with water for injection ( } 2 \mathrm{ml} \\
\text { respules) as placebo delivered intranasally } 2 \text { times a } \\
\text { day for } 3 \text { weeks } \\
+ \text { Oral Doxycycline (antibiotic) tablets } 2 \text { tablets on day } \\
\text { one, followed by one tablet once daily for } 3 \text { weeks }\end{array}$ \\
\hline
\end{tabular}

were on any oral steroid, topical steroid or oral antibiotic in a 6-weeks period prior to enrolment, age less than 18 years, prior sinus surgery history, documented doxycycline or steroid allergy, using CYP450 inhibitors, immunosuppression, uncontrolled diabetes, were all excluded. Patients requesting withdrawal, and treatment non-compliance were removed from the study.

\section{Treatment arms}

Table 1 summarizes the three treatment arms. All patients, blinded to their treatment, administered their oral medications and irrigation solution daily for 3 weeks. Patients were asked to keep a diary and medication packets to be brought back to ensure compliance. At the commencement of the study all patients received treatment packs, consisting of oral medications, FLO sinus care kit with irrigation sachets and an irrigation bottle (to prepare a nasal irrigation solution; active ingredients: sodium chloride, potassium chloride, calcium lactate pentahydrate, sodium bicarbonate, glucose), (ENT technologies, Melbourne, Australia) and vials containing a clear solution to be added to the FLO irrigation solution. Only one of the treatments in the pack had an active medical ingredient, the remaining treatments were placebo. Patients were advised to start the three treatment packs simultaneously.

The active ingredient in arm 1 was oral prednisolone (Aspen Pharmacare, St Leonards, NSW), arm 2 was budesonide, $0.5 \mathrm{mg} / 2 \mathrm{~mL}$ respules (AstraZeneca $A B$, Sodertalje, Sweden) and arm 3 was oral doxycycline (Alphapharm, Millers Point, NSW). The placebo oral medicines were prepared by Professional Pharmaceutical Packaging Pty, Ltd (VIC, Australia) and the placebo for budesonide respules was $2 \mathrm{~mL}$ water for injections (Pfizer, 
Brooklyn, USA). Flo sinus care irrigation was used as the base nasal irrigation for all three groups.

\section{Clinical outcome data}

The patient follow-up was at treatment completion (3-weeks) and at 6-weeks (3-weeks after treatment completion). Patient symptom scoring, endoscopic grading and microbiome swabs were performed on enrolment (day 0), at 3-week (time_3)and at 6-week (time_6). Radiological severity of CRS was scored using the Lund-Mackay score (LMS) ${ }^{(20)}$ prior to recruitment.

Patient symptom scoring included the validated Adelaide disease severity score ${ }^{(21)}$ and Sinonasal outcome test-22 [SNOT22 , minimal clinically important difference (MCID) considered at 8.9] ${ }^{(22)}$. Endoscopic assessment was performed using the validated modified Lund Kennedy scoring ${ }^{(23)}$ which was recorded and then scored in a blinded manner (patient symptom scoring systems described in supplementary data).

\section{Microbiome sample collection}

A guarded flexible nasopharyngeal flocked swab (Copan Italia S.p.A., Brescia, Italy) was rotated 7 times in the middle meatus under endoscopic guidance. The swabs were then transferred into a sterile container, transported on ice and stored at $-80^{\circ} \mathrm{C}$.

\section{DNA extraction}

DNA extraction from swabs, was performed using the Qiagen DNeasy Blood and Tissue kit (Qiagen, Hilden, Germany) with modifications. Briefly, the swabs are held and cut into pieces using sterile forceps and scissors, into a $2 \mathrm{ml}$ microcentrifuge tube containing $180 \mu$ l of Lysozyme (Sigma-Aldrich, St Louis, MI, USA) lysis buffer prepared at a concentration of $20 \mathrm{mg} / \mathrm{ml}$ and left at room temperature overnight. The following day, a $5 \mathrm{~mm}$ stainless steel bead (Qiagen) was added to each tube and beat for 20 seconds at $15 \mathrm{~Hz}$ using Qiagen Tissue Lyser. A 50mg of $0.1 \mathrm{~mm}$ glass bead (Sigma-Aldrich) was added to the tubes after removing steel beads and beat for 5 mins at $30 \mathrm{~Hz}$. To this, $25 \mu \mathrm{l}$ proteinase $\mathrm{K}$ and $200 \mu \mathrm{l}$ Buffer $\mathrm{AL}$ (without ethanol) were added, vortexed and incubated at $56^{\circ} \mathrm{C}$ for 30 mins. The supernatant collected after centrifugation was transferred to a new tube. The protocol was continued according to the manufacturer's instructions and the DNA eluted in 100 ul elution buffer. Samples were quantified using the NanoDrop spectrophotometer (ThermoFisher scientific, MA, USA).

\section{PCR amplification of 16 S rRNA gene and pyrosequencing} Polymerase chain reaction (PCR) amplification and sequencing was performed by the Australian Genome Research Facility. By amplifying the V3 to V4 (341F-806R) hypervariable region of the $16 \mathrm{~S}$ rRNA, gene libraries were generated. PCR amplicons were generated using the primers CCTAYGGGRBGCASCAG in the forward sequence and GGACTACNNGGGTATCTAAT in the reverse sequence, using AmpliTaq Gold 360 Master Mix (Life Technologies, Mulgrave, Australia) using local protocol. Amplicons were measured by fluorometry (Invitrogen Picogreen; Thermo Fisher Scientific, Waltham, MA) and normalized. The equimolar pool was then quantified by quantitative PCR (KAPA Biosystems, Capetown, South Africa) and set up for sequencing on the IIlumina MiSeq (Illumina Inc., San Diego, CA, USA) with 300 base paired end chemistry.

\section{Bioinformatic pipeline}

Demultiplexed fastq files were received from the sequencing facility. We used the new QIIME 2 (version 2018.11) ${ }^{(24)}$ for our bioinformatics pipeline (details in supplementary data). Following taxonomy assignment, mean relative abundance as well as prevalence of the genera were calculated for each group. Shannon's diversity and Faith's phylogenetic diversity index (Faith's PD) (25) were used for alpha diversity. Diversity metrics were generated through sci-kit bio version 0.5.3.

Microbiome stability studies were conducted according to methods described by Martí et al. ${ }^{(26)}$.

\section{Statistical analysis}

Sample size estimation was calculated with alpha $=0.05$, target power $=0 \cdot 80$, and within-subject correlation $=0 \cdot 4$. Considering the preliminary results of unpublished data from our department, we assumed differences of $5-20 \%$ in relative abundance of a taxa in the various treatment arms at different time points, signaling that the taxon was affected by treatment. The power analysis was done using the GLIMMPSE program version 2.1.2 (University of Colorado, Denver, CO, USA). The final sample size was calculated at $\mathrm{n}=45$ ( 15 subjects per group).

We analyzed outcomes using a "Repeated measures ANOVA model without the treatment variable but with the interaction between treatment and time specified as a covariate", as recommended by Twisk et al. for the analysis of RCT outcomes (27) (supplementary data). The study was registered with the Australian New Zealand Clinical Trial Registry (ANZCTR), ACTRN12619001398190.

\section{Results}

\section{Patient cohort}

A total of 178 CRS patients referred to The Queen Elizabeth Hospital, Adelaide between Oct 26, 2016 and Nov 2, 2018, were screened for recruitment. Among these, 69 patients met the eligibility criteria with 50 agreeing for trial enrolment (CONSORT flow chart Figure 1). Following randomization, 5 patients were excluded due to withdrawal or treatment non-compliance. Analysis after unblinding revealed that 1 of these patients was from the active oral steroid, 1 from topical steroid and 3 from the oral antibiotic arm. The remaining 45 patients reported compliance to the 3-week therapy. Table 2 summarizes patient 


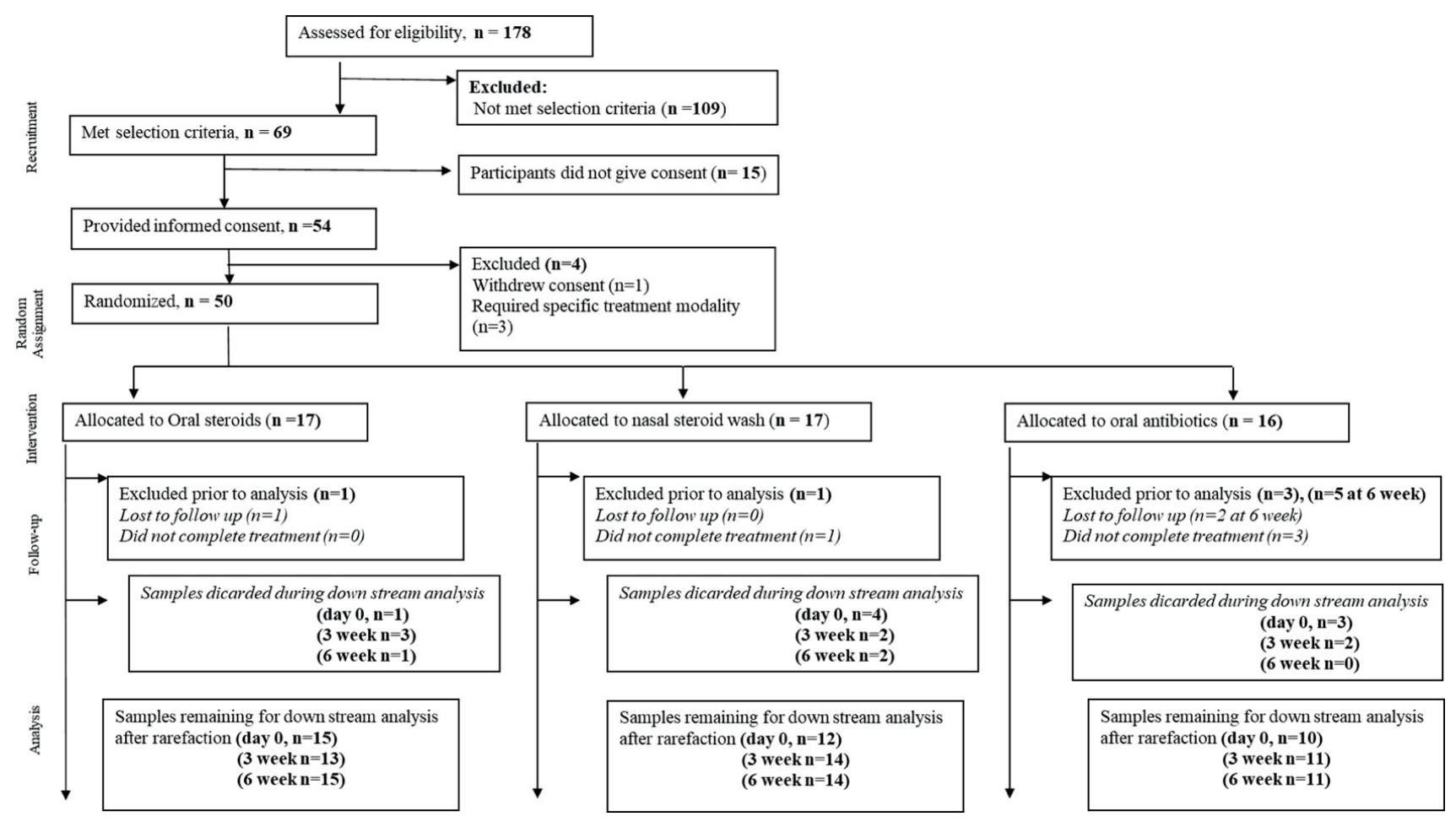

Figure 1. CONSORT flowchart, $n=$ number of patients/samples. "Not meet selection criteria" included patients who were on medications for CRS with in last 6weeks and/or patients not fulfilling EPOS criteria for CRS diagnosis.

Table 2. Baseline characteristics $(n=45)$

\begin{tabular}{|lccc|}
\multicolumn{1}{c}{$\begin{array}{c}\text { Patient } \\
\text { characteristics }\end{array}$} & $\begin{array}{c}\text { Oral } \\
\text { steroid }\end{array}$ & $\begin{array}{c}\text { Topical } \\
\text { steroid }\end{array}$ & $\begin{array}{c}\text { Oral } \\
\text { antibiotic }\end{array}$ \\
\hline Number $(\mathrm{n}=50)$ & $\mathrm{n}=17$ & $\mathrm{n}=17$ & $\mathrm{n}=16$ \\
\hline Mean Age (yrs.) & $44 \cdot 18$ & $41 \cdot 25$ & 39.00 \\
\hline Male/Female & $9 / 7$ & $12 / 4$ & $8 / 5$ \\
\hline CRSsNP/CRSWNP & $10 / 6$ & $8 / 8$ & $10 / 3$ \\
\hline SNOT-22; mean (SD) & $58.4(22.5)$ & $51.7(21.4)$ & $64.1(22.4)$ \\
\hline ADSS; mean (SD) & $14.4(3.0)$ & $13.3(3 \cdot 6)$ & $13.0(2 \cdot 7)$ \\
\hline LKS; mean (SD) & $4.9(2 \cdot 9)$ & $6 \cdot 3(4 \cdot 0)$ & $4.6(3.9)$ \\
\hline LMS; mean (SD) & $12 \cdot 29(4 \cdot 0)$ & $12.94(5.4)$ & $9.56(4 \cdot 0)$ \\
\hline
\end{tabular}

CRSsNP; Chronic rhinosinusitis without polyps, CRSwNP; Chronic rhinosinusitis with polyps, SNOT-22; Sinonasal outcome test 22, ADSS; Adelaide disease severity score, LKS; Lund Kennedy score, LMS; Lund Mackay score, Data are $n$ or mean (SD).

demographics and baseline clinical characteristics. Post-trial follow-up to the 6-week time point was possible for 43 patients with 2 patients, both from the active oral antibiotic arm, lost to follow up.

\section{Clinical outcomes: symptom and endoscopic scores} We observed a decrease in the SNOT-22 and ADSS in all treatment arms at time_3, but a clinically important reduction (>
MCID of 8.9) was observed only in the oral and topical steroid group. The repeated measures model was used for estimation of intra-treatment effect sizes across different timepoints, in comparison to baseline. This revealed a drop (from baseline) of the SNOT-22 mean score of 12.9 [Cl 3.0.22.7] $(p=0.012)$ for prednisolone and 14.4 [Cl 3.9, 24.9] ( $p=0.008)$ for budesonide rinse on treatment completion. In comparison, the time_3 score for doxycycline dropped only $4.6[\mathrm{Cl}-7.4,16.5](p=0.448)$. These improvements were not sustained at the time_ 6 timepoint, with a recurrence towards baseline in all groups. When contrasting the SNOT-22 scores between the three treatment arms at time_3, there was a clinically important difference ( $>$ MCID of 8.9) between prednisolone versus doxycycline (-10-7, $\mathrm{Cl}[-31 \cdot 1$, $9 \cdot 6], p=0.295)$ and between budesonide rinse versus doxycycline $(-17 \cdot 5,[\mathrm{Cl}-38 \cdot 5,3 \cdot 6], \mathrm{p}=0 \cdot 102)$ (Figure 2A).

Changes in the Adelaide Disease Severity Score mirrored that of the SNOT-22 (reductions of 3.0, 3.5, and 1.1 at time_3 for the oral steroid, topical steroid, and oral antibiotic groups, respectively) (Figure 2B).

Similarly, for the Lund-Kennedy scores (LKS), a significant improvement in the scores were observed at time_3 for the prednisolone $(1 \cdot 1[\mathrm{Cl} 0.2,1.9], \mathrm{p}=0.013)$ and the budesonide arms (1.0 $[\mathrm{Cl} 0.1,1.9], \mathrm{p}=0.025)$, while the doxycycline arm scored poorly $(0.3[\mathrm{Cl}-0.7,1.3])$. Again, this improvement in LKS for the first two treatments arms was not sustained at time_6 (Figure 2C) 
A.

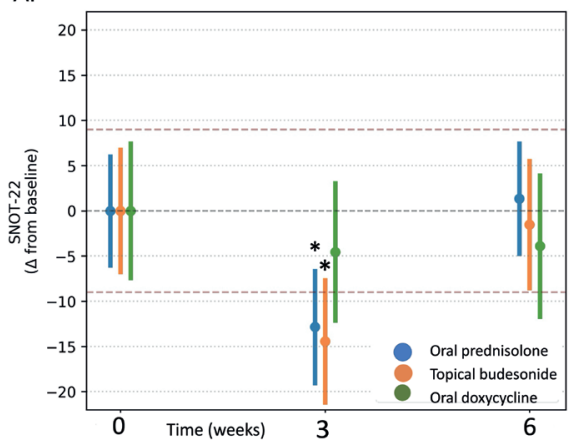

B.

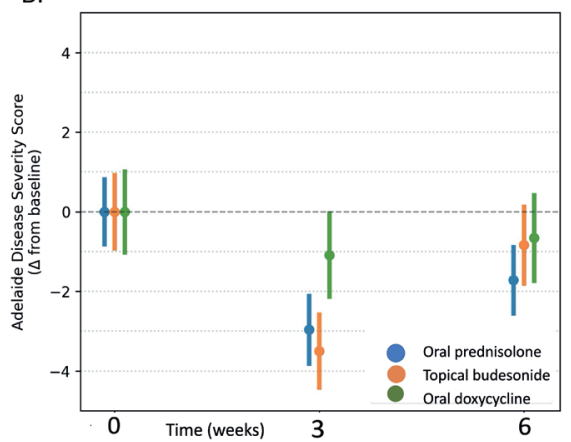

C.

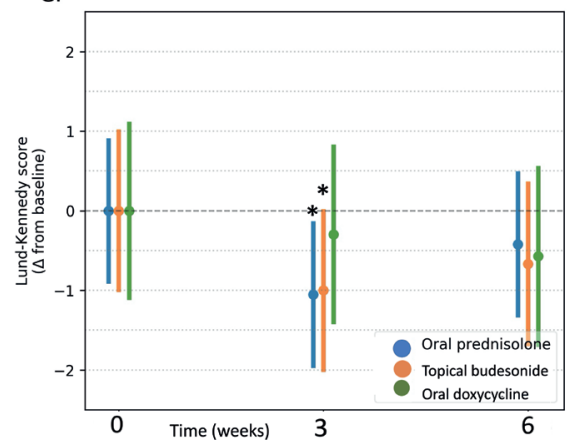

Figure 2. Clinical outcomes of the trial: Data represented as means (circles) and Standard Errors (error bars). SNOT-22 scores (A), Adelaide disease severity scores (ADSS) (B) and Lund-Kennedy scores (C) were inferred from repeated measures model and demonstrated as change from baseline. The maroon dotted line in (A) indicates minimal clinically important difference (MCID). A statistically significant decrease in symptoms scores of SNOT-22, and LKS is seen at 3 weeks in the oral and topical steroid treatment arms. SNOT-22 = Sinonasal outcome test-22; time $0=$ baseline (day 0 ); time $3=$ immediately post-therapy at 3-weeks; time $6=3$-weeks post treatment completion; ${ }^{*}$ represent $\mathrm{p}$ - value $<0.05$.

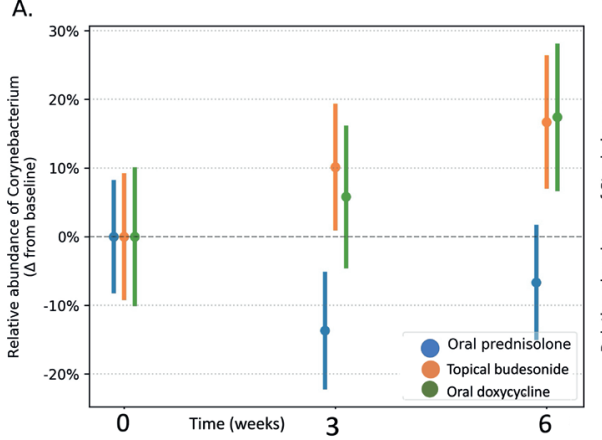

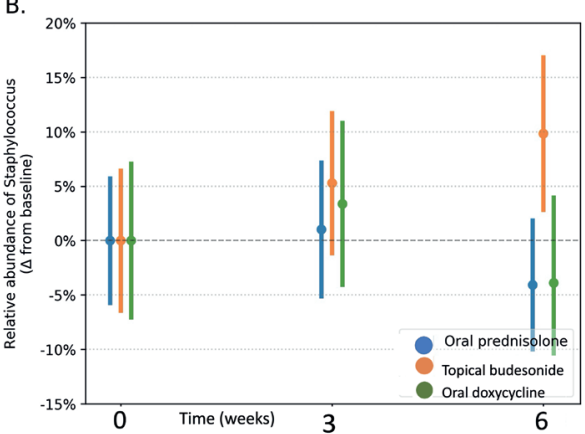

Figure 3. Relative abundance of Corynebacterium and Staphylococcus, showing an increasing Corynebacterium with topical budesonide and oral doxycycline and decreasing Corynebacterium with oral prednisolone at 3 and 6-weeks. Data represented as means (circles) and Standard Errors (error bars). Relative abundance of Corynebacterium (A) and of Staphylococcus (B), as inferred from repeated measures model, demonstrated as change from baseline.

\section{Microbiome outcomes: taxonomy}

Next, we assessed the differential relative abundance trends across the follow-up timepoints with different treatments. Corynebacterium and Staphylococcus were the most common genera in all groups at all assessed time points. Although this showed some trends for the major taxa with different treatments, no single comparison was statistically significant at the 0.05 level (Figure 3A \&B).

Minimal non-significant decrease in relative abundance of Corynebacterium -6.6 [Cl 7.9,-21.2] $(\mathrm{p}=0.36)$ and Staphylococcus were observed in patients receiving prednisolone at time_6. In patients randomised to the budesonide irrigation, there was a tendency towards a gradual increase in the relative abundance of Corynebacterium and Staphylococcus at time_3 and time_6. At time_6, the increase in relative abundance was $16 \cdot 7 \%[\mathrm{Cl}-0 \cdot 3$, $33.8](p=0.055)$ for Corynebacterium and 9.8\% [Cl-7.2, 26.8] ( $p=$ $0 \cdot 252$ ) for Staphylococcus compared to baseline (Figure 3A \& B). With antibiotics, a tendency towards a gradual increase in the relative abundance of Corynebacterium was observed, reaching $17.4 \%[\mathrm{Cl}-1.6,36.4](p=0.071)$ increase at time_6 compared to baseline. Minimal non-significant reductions in relative abundance of Staphylococcus were observed in those patients at time_6, compared to baseline.

\section{Microbiome outcomes: diversity and stability studies} We measured Faith's PD and Shannon's index as measures of phylogenetic and non-phylogenetic alpha diversity, respectively to assess the microbiome diversity outcomes. Budesonide rinses increased Faith's PD at time_3, (a $1 \cdot 1[\mathrm{Cl}$ 0.1, 2.1] increase, compared to baseline; $p=0.031$ ), but with a rebound towards baseline value at time_6. No statistically significant changes were observed in Shannon's index, despite a trend towards decreasing diversity at time_6 with antibiotic treatment $(-0 \cdot 6, \mathrm{Cl}$ $[-1.4,0 \cdot 1], p=0.094$ ) (Figure 4A \& B).

We used Rank variability (RV) and Differences variability (DV) as surrogates for assessing microbiome stability ${ }^{(26)}$. When a large number of bacterial taxa change their ranking in terms of abundance compared to the average, the RV and DV become higher (and the microbiome becomes, supposedly, more labile i.e. less stable). In our trial, the oral and topical steroid arms demon- 
A.
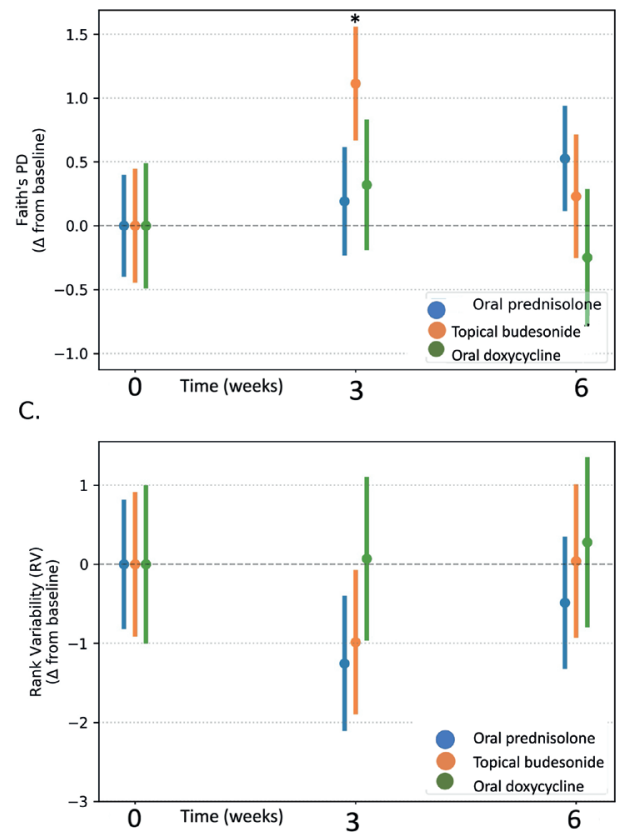

B.

D.
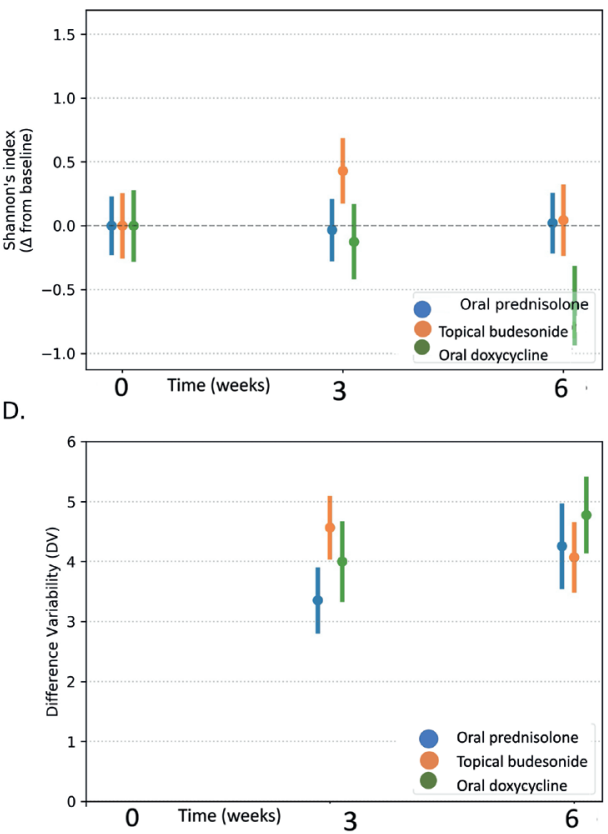

Figure 4. Microbiome alpha-diversity and stability indices. Data represented as means (circles) and Standard Errors (error bars). Faith's phylogenetic diversity index (Faith's PD) (A), Shannon's index (B), Rank Variability (RV) (C) as inferred from repeated measures model, and Difference Variability (DV) (D) raw scores. A statistically significant increase in bacterial diversity is seen Faith's PD with topical budesonide. Time $0=$ baseline (day 0 ); time $3=$ immediately post-therapy at 3 -weeks; time $6=3$-weeks post treatment completion; ${ }^{*}$ represent $\mathrm{p}$ - value $<0.05$.

strated a (temporary) reduction in RV at time_3. Comparatively speaking, antibiotic treatment had higher RV and the highest DV at all follow-up timepoints, albeit all RV and DV comparisons were not statistically significant at the 0.05 level and it should be interpreted with caution.

\section{Subgroup analyses: polyps versus non-polyps}

To investigate the effect of nasal polyps on outcomes, we conducted separate repeated measures models for the CRSsNP and CRSwNP subgroups. The improvement in SNOT-22 scores for the CRSsNP at time_3 compared to baseline were: $11 \cdot 0[\mathrm{Cl}-1.9,23.9]$ for the oral steroid; $7.9[\mathrm{Cl}-6 \cdot 1,21 \cdot 8]$ for the topical steroid; and $6.6[\mathrm{Cl}-6.9,20.0]$ for the oral antibiotic. On the other hand, the improvement at time_3 was more pronounced in the CRSwNP for the oral steroid $(15.8[\mathrm{Cl}-0.8,32.4], \mathrm{p}=0.06)$ and the topical steroid groups $(21.3[\mathrm{Cl} 5 \cdot 1,37 \cdot 6], \mathrm{p}=0.013)$, while the antibiotic had an overall average worsening of symptoms at time_3 (-4.6 $[\mathrm{Cl}-30 \cdot 1,21 \cdot 0], \mathrm{p}=0.714)$. Again, a rebound to baseline was observed for both steroid groups at time_6. (Figure 5A-1 \& B-1). Figure 5 also shows that, at least in our cohort, there appeared to be no consistent correlation of the symptomatic outcome with the microbiomic parameters in all the treatments at any particular time point (relative abundance of Corynebacterium and Staphylococci; Figure 5A-11, B11 \& A-111, B-111 and Faith's PD; Figure 5A1V, B1V).

\section{Discussion}

Previous studies that attempted to define the healthy and diseased sinonasal microbiota have shaped a present day understanding of the role of "dysbiosis" in CRS. Our study takes this a step further by attempting to understand microbiome changes and their association with the clinical outcomes of the currently recommended medical therapies in CRS.

We observed an immediate post-treatment significant improvement in the subjective (SNOT-22 \& ADSS) and objective (LKS) clinical scores of CRS patients treated with either oral or topical steroids for 3 weeks and not with doxycycline. The improvement observed with steroid treatment was not sustained however, with the clinical scores returning to base line, 3 weeks post treatment cessation. Subgroup analysis based on phenotype suggests that the greatest clinical benefit in the steroid arms was seen in CRSwNP, which is an expected finding. Unexpectedly however, was the apparent worsening of symptoms in CRSwNP treated with doxycycline, despite its often-referenced local antiinflammatory action (specifically chosen for this study based on this action) and broad-spectrum anti-bacterial activity ${ }^{(28,29)}$. Another interesting finding of this study was the lack of significant alteration in the microbiomes with the different treatments, despite clinical changes observed. Both these observations do lend support to the hypothesis that the inflammation seen in CRS is non-infective. Although the choice of doxycycline and its use empirically without first obtaining a culture, may also 

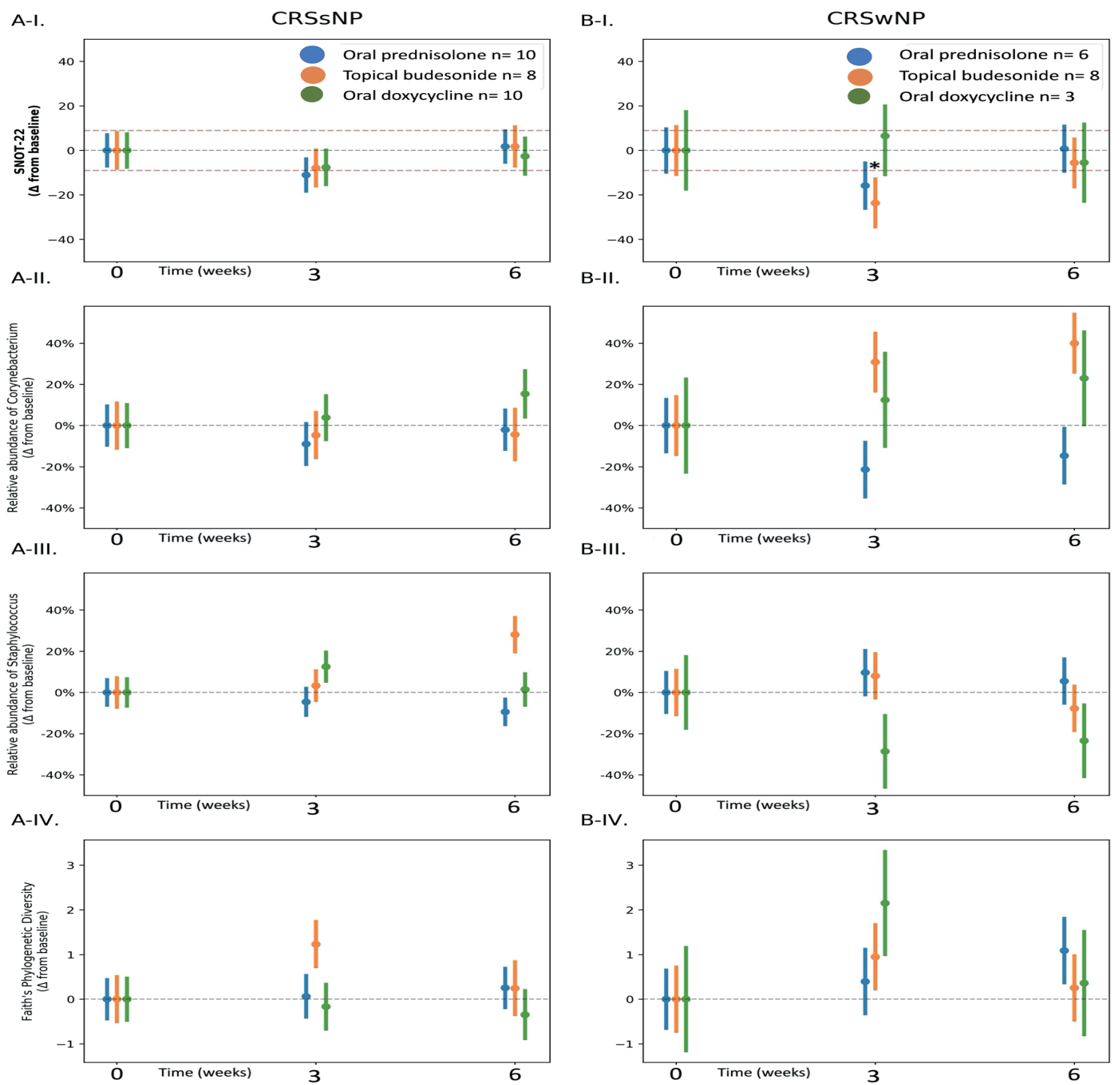

B-IV

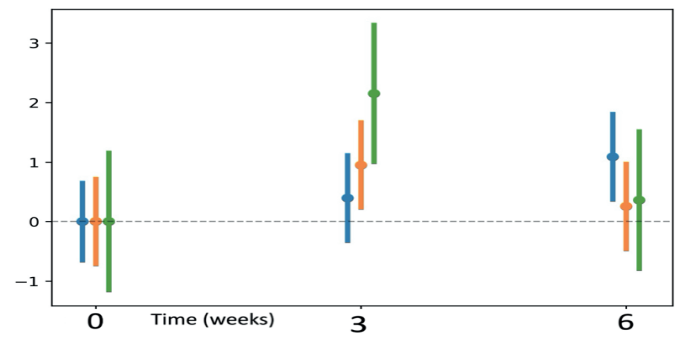

Figure 5. Subgroup analysis of clinical and microbiomic indices: Subgroup analysis demonstrating SNOT 22 score improvement on treatment with oral and topical steroids, and a worsening with oral antibiotics in CRSwNP patients at 3 weeks. A statistically significant decrease in the clinical symptoms on SNOT-22 is seen with topical budesonide. Data represented as means (circles) and Standard Errors (error bars) and the results inferred from a repeated measures model. The maroon dotted line indicates minimal clinically important difference (MCID) (14). SNOT-22 = Sinonasal outcome test-22; CRSsNP= Chronic rhinosinusitis without polyps; CRSwNP= Chronic rhinosinusitis with polyps; Faith's PD = Faith's phylogenetic diversity index. Time 0 = baseline (day 0 ); time $3=$ immediately post-therapy at 3 weeks; time $6=3$-weeks post treatment completion; ${ }^{*}$ represent $p$ - value $<0.05$.

explain the above results and could be considered a limitation of this study, the study design was deliberate in its attempt to replicate common treatment practices of clinicians.

High level evidence supports the use of both oral and topical steroids in the treatment of CRSWNP ${ }^{(5,19)}$. In CRSwNP oral steroids have been shown to reduce the inflammatory markers, radiological and endoscopic scores, improving patient symptoms on an immediate ( 2 week) and longer follow up (12 weeks) ${ }^{(5,}$ ${ }^{30,31)}$. A systematic review on topical steroid use for CRSwNP by Rudmick et al. ${ }^{(32)}$ demonstrated consistent symptoms reduction with the use of mometasone, fluticasone, and budesonide.

The evidence for steroid use in CRSsNP is less clear with a recent meta-analysis by Kalish et al. ${ }^{(33)}$ failing to show overall benefit. However, their use appeared safe and with symptomatic benefit (demonstrated in few studies) leading the authors to conclude that they are a reasonable treatment option for CRSsNP. Oral steroids in CRSsNP is not sufficiently supported in literature ${ }^{(31)}$ and given the higher side effect profile, is recommended against for this phenotype.

In our trial, budesonide rinses in unoperated patients showed comparable clinical benefits to oral steroids. This was also observed in our subgroup analysis of CRSwNP. Although not approved by the U.S food and drug administration (FDA) for use in CRS ${ }^{(19)}$, topical budesonide irrigations remain common practice 
throughout the world particularly post-surgery. Their use is supported by safety studies that demonstrate no significant effect on the hypothalamic-pituitary-axis or intraocular pressure ${ }^{34,}$ ${ }^{35)}$. Furthermore, double blinded placebo-controlled trials have consistently demonstrated benefits with budesonide rinses ${ }^{34,}$ ${ }^{36,37)}$. Our study's findings add to the ever-growing body of literature that budesonide irrigations may reduce the need for oral corticosteroids with potential systemic side effects despite their FDA approval for CRSwNP.

Antibiotics continue to be used in the management of CRS despite its low level of evidence. Based on our findings, as well as the risk of side effects and the ever-increasing issue of antibiotic resistance we do not recommend empirical antibiotics as routine in treatment of CRS contradicting previous observations $(19,29)$.

Unique to this study was the analysis of treatment effects on the sinonasal microbiome. Consistent with previous literature, Corynebacterium and Staphylococcus taxa were the most abundant ${ }^{(13,38)}$. Interestingly, although trends to microbiome change were observed in all treatment groups, statistical significance was not achieved. This may reflect a lack of significant effect of treatments on the local sinonasal microbiome or could be that the study was underpowered to determine a significant difference or could be due to the inability of current bioinformatic technology to accurately speciate bacteria. A significant microbial finding of this study was the increased diversity seen immediately after pulmicort treatment. Microbiome diversity is generally associated with health in other body systems ${ }^{(39)}$. Similar to the clinical improvement in this cohort, this increase in diversity also reduced back to pre-treatment levels after treatment cessation. Although not statistically significant, treatment with doxycycline demonstrated the converse, with reduced bacterial diversity immediately after treatment. This antibiotic related effect is reported in other areas of medicine and is likely due to the selective pressures on bacterial growth associated with antimicrobial use ${ }^{(40)}$. Interestingly, in our study, worsening of clinical scores seemed to correspond to the reduction in bacterial diversity. The above findings raise the possibility that bacterial diversity may be more important in determining health than the specific bacteria, given that the latter did not appear to change significantly with treatment.

A relatively new concept in healthy and diseased is microbial temporal stability or variability over time ${ }^{(26)}$. Their study found statistically increased microbial variability for subjects taking antibiotics, and patients with inflammatory bowel disease. In our results, the RV and DV indices of microbial stability could suggest (albeit not statistically significant) the potential development of a progressively unstable microbiome with continued oral antibiotic use. These results were associated with a lower Shannon's diversity index in the antibiotic group.
Limitations: Sample size was an obvious limitation of our study. The strict inclusion criteria eliminated patients currently on, or who had recently been treated with medical therapie, aiming to remove the possible confounding effect of concurrent treatments on the clinical and microbial changes in our cohort. As most patients receive a trial of medical therapy from their primary care physicians prior to a tertiary rhinology center referral, this significantly reduced the number of patients eligible for study inclusion, and consequently effected its power.

Other limitations include the absence of a saline nasal irrigation arm (which could potentially influence clinical and microbiome outcomes $\left.{ }^{(14,41,42)}\right)$, absence of a visual analogue score to assess disease severity and absence of inflammatory endotyping of CRS patients.

\section{Conclusion}

We can conclude that steroids (topical and oral) provide symptomatic improvement, in CRS patients, only as long as they continue to be given. Although not FDA approved, topical corticosteroids seemed to be as effective as oral prednisolone even in the unoperated patient. This may have significant implications in the maximal medical treatment of patients prior to surgery, as evidence is emerging that steroid delivered topically may have less systemic side effects than oral steroids.

\section{Acknowledgements}

We thank all the participants of this trial and the outpatient department staff for their enthusiastic involvement. This study is supported the Garnett Passe and Rodney Williams memorial foundation conjoint grant.

The Garnett Passe and Rodney Williams memorial foundation conjoint grant to AJP and SV; The Hospital Research Foundation, Woodville, Australia; L.C. has received the Adelaide Scholarship International, University of Adelaide, Adelaide, Australia

\section{Authorship contribution}

LMC contributed to the protocol development and study design, did data collection, coordinated the study, did primary data analysis, wrote the initial draft of the manuscript. $A B$ did the data analysis and interpretation, contributed to the study design and protocol and provided inputs on subsequent manuscript drafts. CC contributed to the data collection and provided inputs to the manuscripts. SV and PJW and AP contributed to the study design and protocol, and critically revised the manuscript. AP contributed to the study design and protocol and was involved in significant writing of the manuscript. All authors have read and approved the final version of the manuscript.

\section{Conflict of interest}

None provided. 


\section{References}

1. Lam K, Schleimer R, Kern R. The Etiology and Pathogenesis of Chronic Rhinosinusitis: a Review of Current Hypotheses. Curr Allergy Asthma Rep. 2015; 15:1-10.

2. Brook I. Microbiology of chronic rhinosinusitis. Eur J Clin Microbiol Infect Dis . 2016 35:1059-1068.

3. Wagner Mackenzie B, Waite DW, Hoggard M, Douglas RG, Taylor MW, Biswas K. Bacterial community collapse: a meta-analysis of the sinonasal microbiota in chronic rhinosinusitis. Environ Microbiol. 2017 19:381- 392.

4. Hoggard M, Wagner Mackenzie B, Jain R, Taylor MW, Biswas K, Douglas RG. Chronic Rhinosinusitis and the Evolving Understanding of Microbial Ecology in Chronic Inflammatory Mucosal Disease. Clin Microbiol Rev. 2017; 30:321-348.

5. Orlandi RR, Kingdom TT, Hwang PH, et al. International Consensus Statement on Allergy and Rhinology: Rhinosinusitis Executive Summary. Int Forum Allergy Rhinol. 2016; 6 Suppl 1:S3-21.

6. Hissaria P, Smith W, Wormald PJ, et al. Short course of systemic corticosteroids in sinonasal polyposis: A double-blind, randomised, placebo-controlled trial with evaluation of outcome measures. J Allergy Clin Immunol. 2006; 118:128-133.

7. Rhen T, Cidlowski JA. Antiinflammatory Action of Glucocorticoids - New Mechanisms for Old Drugs. N Engl J Med. 2005; 353:1711-1723.

8. Cherian LM, Cooksley C, Richter K, et al. Effect of commercial nasal steroid preparation on bacterial growth. Int Forum Allergy Rhinol. 2019; 9:766-775.

9. Abreu NA, Nagalingam NA, Song $Y$, et al. Sinus microbiome diversity depletion and Corynebacterium tuberculostearicum enrichment mediates rhinosinusitis. Sci Transl Med. 2012; 4:151ra124

10. Cleland EJ, Bassiouni A, Vreugde S, Wormald P-J. The bacterial microbiome in chronic rhinosinusitis: Richness, diversity, postoperative changes, and patient outcomes. Am J Rhinol Allergy. 2016; 30:37-43

11. Carlson-Jones JA, Paterson JS, Newton K, et al. Enumerating virus-like particles and bacterial populations in the sinuses of chronic rhinosinusitis patients using flow cytometry. PLoS One 2016;11: e0155003.

12. Hox V, Lourijsen E, Jordens A, et al. Benefits and harm of systemic steroids for short- and long-term use in rhinitis and rhinosinusitis: an EAACI position paper. Clin Transl Allergy. 2020; 10:1-27.

13. Jain R, Hoggard $M$, Zoing $M$, et al. The effect of medical treatments on the bacterial microbiome in patients with chronic rhinosinusitis: a pilot study. Int Forum Allergy Rhinol. 2018; 8:890-899

14. Liu CM, Kohanski MA, Mendiola M, et al. Impact of saline irrigation and topical corticosteroids on the postsurgical sinonasal microbiota. Int Forum Allergy Rhinol. 2015; 5:185-90.
15. Barshak MB, Durand ML. The role of infection and antibiotics in chronic rhinosinusitis. Laryngoscope Investig Otolaryngol. 2017; 2:36-42.

16. Smith SS, Evans CT, Tan BK, Chandra RK, Smith SB, Kern RC. National burden of antibiotic use for adult rhinosinusitis. J Allergy Clin Immunol. 2013; 132:1230-1232.

17. Ajitha T, Paul K. Neonates, antibiotics and the microbiome. Nat Med. 2014; 20:469-470.

18. lizumi T, Battaglia T, Ruiz V, Perez Perez Gl. Gut Microbiome and Antibiotics. Arch Med Res. 2017; 48:727-34.

19. Fokkens WJ, Lund VJ, Mullol J, et al. EPOS 2012: European position paper on rhinosinusitis and nasal polyps 2012. A summary for otorhinolaryngologists. Rhinology. 2012 50:1-12.

20. Lund VJ, Mackay IS. Staging in rhinosinusitus. Rhinology. 1993; 31:183-184.

21. Naidoo Y, Tan N, Singhal D, Wormald PJ. Chronic rhinosinusitis assessment using the Adelaide Disease Severity Score. J Laryngol Otol. 2013; 127:S24-28.

22. Hopkins C, Gillett S, Slack R, Lund VJ, Browne JP. Psychometric validity of the 22-item Sinonasal Outcome Test. Clin Otolaryngol. 2009; 34:447-454

23. Psaltis AJ, Li G, Vaezeafshar R, Cho KS, Hwang PH. Modification of the LundKennedy endoscopic scoring system improves its reliability and correlation with patient-reported outcome measures. Laryngoscope. 2014; 124:2216-2223

24. Bolyen E, Dillon M, Bokulich N, et al. QIIME 2: Reproducible, interactive, scalable, and extensible microbiome data science. PeerJ Prepr. 2018; 6:e27295v2

25. Faith DP. Conservation evaluation and phylogenetic diversity. Conserv Biol. 1992; 61:110.

26. Jose Manuel M, Daniel M-M, Teresa R, et al. Health and Disease Imprinted in the Time Variability of the Human Microbiome. mSystems. 2017; 2:e00144-16.

27. Twisk J, Bosman L, Hoekstra T, Rijnhart J, Welten M, Heymans M. Different ways to estimate treatment effects in randomised controlled trials. Contemp Clin Trials. 2018; 10:80-85.

28. Di Caprio R, Lembo S, Di Costanzo L, Balato A, Monfrecola G. Anti-Inflammatory Properties of Low and High Doxycycline Doses: An Study. Mediators Inflamm. 2015; 2015:10

29. Van Zele T, Gevaert P, Holtappels G, et al Oral steroids and doxycycline: Two different approaches to treat nasal polyps. J Allergy Clin Immunol. 2010; 125:1069-1076.

30. Benítez P, Alobid I, De Haro J, et al. A Short Course of Oral Prednisone Followed by Intranasal Budesonide Is an Effective Treatment of Severe Nasal Polyps. Laryngoscope. 2006; 116:770-775.

31. Guilemany JM, Alobid I, Mullol J. Controversies in the treatment of chronic rhinosinusitis. Expert Rev Respir Med. 2010; 4:463-477.

32. Rudmik L, Schlosser RJ, Smith TL, Soler
ZM. Impact of topical nasal steroid therapy on symptoms of nasal polyposis. Laryngoscope. 2012; 122:1431-1437.

33. Kalish LH, Arendts G, Sacks R, Craig JC. Topical Steroids in Chronic Rhinosinusitis Without Polyps: A Systematic Review and Meta-Analysis. Otolaryngol Head Neck Surg. 2009; 141:674-683.

34. Rotenberg BW, Zhang I, Arra I, Payton KB. Postoperative care for Samter's triad patients undergoing endoscopic sinus surgery: A double-blinded, randomised controlled trial. Laryngoscope. 2011; 121:27022705.

35. Smith KA, French G, Mechor B, Rudmik L. Safety of long-term high-volume sinonasal budesonide irrigations for chronic rhinosinusitis. Int Forum Allergy Rhinol. 2016; 6:228-232.

36. Snidvongs K, Pratt E, Chin D, Sacks R, Earls P, Harvey RJ. Corticosteroid nasal irrigations after endoscopic sinus surgery in the management of chronic rhinosinusitis.(Report). Int Forum Allergy Rhinol. 2012; 2: 415-421

37. Tait S, Kallogjeri D, Suko J, Kukuljan S, Schneider J, Piccirillo JF. Effect of Budesonide Added to Large-Volume, Lowpressure Saline Sinus Irrigation for Chronic Rhinosinusitis: A Randomised Clinical Trial. JAMA Otolaryngol Head Neck Surg. 2018; 144:605-612.

38. Hoggard $M$, Biswas $K$, Zoing $M$, et al. Evidence of microbiota dysbiosis in chronic rhinosinusitis. Int Forum Allergy Rhinol. 2017; 7:230-239.

39. Structure, function and diversity of the healthy human microbiome. Nature. 2012; 486:207-214.

40. Langdon A, Crook N, Dantas G. The effects of antibiotics on the microbiome throughout development and alternative approaches for therapeutic modulation. Genome Med. 2016; 8:39

41. Luk LJ, Delgaudio JM. Topical Drug Therapies for Chronic Rhinosinusitis. Otolaryngol Clin North Am. 2017; 50: 533543.

42. Hauser LJ, Ir D, Kingdom TT, Robertson CE, Frank DN, Ramakrishnan VR. Evaluation of bacterial transmission to the paranasal sinuses through sinus irrigation. Int Forum Allergy Rhinol 2016; 6:800-806

Associate Professor Alkis J. Psaltis

Department of Otolaryngology

Head and Neck Surgery

Queen Elizabeth Hospital Adelaide-

South Australia

Department of Surgery

University of Adelaide

Woodville South, SA 5011

Australia

E-mail: alkis.psaltis@adelaide.edu.au 


\section{SUPPLEMENTARY DATA}

\section{Materials and methods}

Patient clinical scores:

Lund-Mackay score: This is a scoring of the computer tomography of the paranasal sinuses wherein each sinus group is scored as 0,1 or 2 (no abnormality, partial opacification or total opacification). The ostiomeatal complex is scored as "0" (not obstructed) or "2" (obstructed). The score range is $0-24$.

Adelaide disease severity score: This scores 5 most significant sinonasal symptom of chronic rhinosinusitis. Each symptom is scored on a scale of 1 to 5 . This also contained a general quality of life visual analogue score on a scale in the range 0 to 7 .

Sinonasal outcome test: This is a patient reported measure with a broad range of health and health -related quality of life questions used in chronic rhinosinusitis. The scores range from a minimum of 0 to maximum of 110. To meaningfully interpret the clinical importance of the differences in the measures of SNOT-22 within groups and individuals, the smallest change in scores that a group of patients can detect as real improvement (minimally important difference-MID) is calculated. The MID was taken at 8.9 in our study.

Modified Lund-Kennedy score: This is a scoring of the rigid nasal endoscopy for polyps, odema and discharge, each graded as 0,1 or 2 . The scores thus ranged from 0 to 6 .

\section{Bioinformatics}

Forward and reverse reads were joined using PEAR ${ }^{(1)}$ through the QIIME 2 plugin q2-pear (https://github.com/bassio/q2-pear). Joined sequences were then quality-filtered using the QIIME 2 plugin q2-quality-filter (2), with minimum quality parameter of $20^{(3)}$. Denoising and Amplicon Sequence Variant (ASV) formation were done using deblur ${ }^{(3)}$ through the q2-deblur plugin with setting "trim-size" $=435$ and with otherwise default parameters. Taxonomy assignment was done against the Greengenes $16 \mathrm{~S}$ reference database (the $99 \%$ clustered similarity sequences) ${ }^{(4)}$ version 13.8 (August 2013) using the BLAST-based classifier implemented in QIIME 2 (q2-feature-classifier) ${ }^{(5)}$ and which implements a Lowest Common Ancestor (LCA) consensus algorithm. The SATé-enabled phylo- genetic placement (SEPP) technique ${ }^{(6)}$ was used for insertion of the ASVs into the high-quality tree generated from the $99 \%$ OTUs Greengenes reference database.

A rarefaction depth cut-off was chosen at 400 before downstream analysis. Taxa were compared at the genus level with additional specieslevel analyses for Staphylococcus aureus and epidermidis. The taxonomic assignment of the two DNA-negative control samples containing extraction reagents only was explored. The bacterial genus Flavobacterium was common to both samples and was present in relatively low abundance in many samples, so this Microbiome stability studies were conducted according to methods described by Martí et al. Rank variability (RV) is a per-sample index, and a surrogate for microbiome stability. It is defined by Martí et al. as "the absolute difference between each taxon rank and the overall rank" (7). Differences variability (DV) is another index defined as "the absolute difference between each taxon rank at a given time and the value it had in the previous time step, averaged over all taxa present $^{\prime \prime(7)}$. These were calculated using a Python implementation of the equations described in the original paper ${ }^{(7)}$ genus was excluded before downstream statistical analyses.

\section{Statistical analysis}

We analyzed outcomes using a "Repeated measures ANOVA model without the treatment variable but with the interaction between treatment and time specified as a covariate", as recommended by Twisk et al for the analysis of RCT outcomes ${ }^{(8)}$. Estimated marginal means, standard errors (SEs), 95\% confidence intervals, and contrasts between study groups were extracted from model results using the R package "emmeans" (9). For all models mentioned, a linear mixed modelling approach was used (through the R packages Ime4 and ImerTest) ${ }^{(10,11)}$ to adjust for the dependency of repeated observations within each patient enrolled in the trial by specifying the patient variable as a random effect, following the paper of Twisk et al. ${ }^{(8)}$. All statistical analyses were performed using $R^{(12)}$ (R Foundation for Statistical Computing, Vienna, Austria) and the Python scientific stack ${ }^{(13)}$ through the Jupyter notebook interface ${ }^{(14)}$.

\section{References}

1. Zhang J, Kobert K, Flouri T, Stamatakis A. PEAR: fast and accurate Illumina Paired-End reAd mergeR. Bioinformatics. 2014:30:614-20.

2. Nicholas $A B$, Sathish $S$, Jeremiah JF, et al. Qualityfiltering vastly improves diversity estimates from Illumina amplicon sequencing. Nat Methods. 2012;10:57.

3. McDonald D, Navas-Molina J, Morton J, et al. Deblur Rapidly Resolves Single-Nucleotide Community Sequence Patterns. MSystems. 2017;2(2):e00191-16.

4. DeSantis TZ, Hugenholtz P, Larsen N, et al. Greengenes, a Chimera-checked 16S rRNA gene database and workbenchcompatible with ARB. Appl Environ Microbiol. 2006;72(7):5069-72

5. Nicholas AB, Benjamin DK, Jai Ram R, et al.
Optimizing taxonomic classification of markergene amplicon sequences with QIIME 2's q2-feature-classifier plugin. Microbiome. 2018;6:1-17.

6. Stefan J, Daniel M, Antonio G, et al. Phylogenetic placement of exact amplicon sequences improves associations with clinical information. mSystems. 2018;3:e00021-18.

7. Jose Manuel M, Daniel Min-Miin, Teresa R, Cííésar G, Manuel Piiena, Amparo L, et al. Health and Disease Imprinted in the Time Variability of the Human Microbiome. mSystems. 2017;2:e00144-16.

8. Twisk J, Bosman L, Hoekstra T, Rijnhart J, Welten M Heymans M. Different ways to estimate treatment effects in randomised controlled trials. Contemp Clin Trials Commun. 2018;10:80-5.

9. R. L. Emmeans: Estimated Marginal Means, aka Least-Squares Means. 2018
10. Douglas B, Martin M, Ben B, Steve W. Fitting Linear Mixed-Effects Models Using Ime4. J Statist Soft. 2015;67:1-48

11. Alexandra K, Per BB, Rune HBC. ImerTest Package: Tests in Linear Mixed Effects Models. J Statist Soft. 2017;82:1-26.

12. R Core Team. R: A Language and Environment for Statistical Computing. Vienna ARFfSC. 2018.

13. Oliphant TE. Python for Scientific Computing Computing in Science \& Engineering. 2007:9:1020.

14. Positioning and Power in Academic Publishing: Players, Agents and Agendas: Proceedings of the 20th International Conference on Electronic Publishing. Ringgold, Inc.; 2016. 